EESTI NSV TEADUSTE AKADEEMIA TOIMETISED. IX KÖIDE FOOSIKALIS-MATEMAATILISTE JA TEHNILISTE TEADUSTE SEERIA. 1960, NR. 4

ИЗВЕСТИЯ АКАДЕМИИ НАУК ЭСТОНСКОИ ССР. ТОМ ІХ СЕРИЯ ФИЗИКО-МАТЕМАТИЧЕСКИХ И ТЕХНИЧЕСКИХ НАУК. 1960, № 4

\title{
О ПОСТРОЕНИИ ПРОИЗВЕДЕНИИ ДВУХ ПЕРЕСТАНОВОЧНЫХ ГРУПП
}

\section{и. ПЕТЕРСЕН}

Назовем группу $G$ косым произведением двух своих подгрупп $A$ и $B$ и обозначим $G=A \circ B$, если всякий элемент $g$ группы $G$ может быть однозначно представлен в виде $g=a b$, где $a \in A, b \in B$. Из этого определения следует $A B=B A$ и $A \cap B=e$. Г. Саппа показал ['], что всякое косое произведение $A \circ B$ можно представить как множество всех пар $[a, b]$ с операцией

$$
\left[a_{1}, b_{1}\right]\left[a_{2}, b_{2}\right]=\left[a_{1} a_{2}^{b_{1}}, b_{1}^{a_{2}} b_{2}\right],
$$

тде элементы $a_{2}^{b_{1}} \in A$ и $b_{1}^{\tau_{2}} \in B$ однозначно определены элементами $a_{2}$ и $b_{1}$ и соответствия $a_{2} \longleftrightarrow a_{2}^{b_{1}}$ и $b_{1} \leftrightarrow b_{1}^{a_{2}}$ являются взаимно однозначными.

Условия

$$
\begin{gathered}
\left(a^{b_{1}}\right)^{b_{2}}=a^{b_{2} b_{1}}, \quad\left(b^{a_{1}}\right)^{c_{2}}=b^{a_{1} a_{2}}, \\
\left(a_{1} a_{2}\right)^{b}=a_{1}^{b} a_{2}^{b a_{1}},\left(b_{1} b_{2}\right)^{a}=b_{1}^{a^{b_{2}}} b_{2}^{a}
\end{gathered}
$$

необходимы и достаточны, чтобы операция (1) была групповой.

В настоящей заметке исследуется построение всех таких косых произведений двух групп $A$ и $B$, один множитель (например $B$ ) которых не содержит нетривиального нормального делителя произведения. Построение таких произведений сводится к построению изоморфных одному множителю $(B)$ подгрупп определенного типа группы всех взаимно однозначных отображений другого множителя $(A)$.

Назовем взаимно однозначное отображение $\varphi$ группы $A$ на себя $e$-о т о бр а жен и ем, если оно оставляет единицу $e$ группы $A$ на месте: $e \varphi=e$. Если $\varphi e$-отображение и $a \in A$, то и отображение, переводящее элемент $x \in A$ в элемент $(a \varphi)^{-1}(a x) \varphi$, является $е$-отображением. Назовем это отображение, следуя Дугласу [2], т р а н с л ц и е й отображения 4 элементом $a$ и обозначим $\varphi T_{a}$, так что

$$
x \varphi T_{a}=(a \varphi)^{-1}(a x) \varphi .
$$

Очевидно, автоморфизмы группы $A$ и только они остаются на месте при трансляции всеми элементами группы $A$. 
Имеют место равенства

$$
\begin{aligned}
\left(a_{1} a_{2} \cdots a_{n}\right) \varphi & =a_{1} \varphi \cdot a_{2} \varphi T_{a_{1}} \cdots a_{n} \varphi T_{a_{1} a_{y}} \cdots a_{n-1}, \\
\left(\varphi_{1} \varphi_{2} \cdots \varphi_{n}\right) T_{a} & =\varphi_{1} T_{a} \cdot \varphi_{2} T_{a \varphi_{1}} \cdots \varphi_{n} T_{a_{\varphi_{1} \varphi_{2}} \cdots \vartheta_{n-1}}, \\
\left(\varphi T_{a_{1}}\right) T_{a_{2}} & =\varphi T_{c_{1} a_{2}} .
\end{aligned}
$$

Действительно, если в (4) взять $a=a_{1}, x=a_{2}$, то имеем $\left(a_{1} a_{2}\right) \varphi=$ $=a_{1} \varphi \cdot a_{2} \varphi T_{a}$, откуда по индукции получим (5). Если же в (4) взять $\varphi=\varphi_{1} \varphi_{2}$, то

$$
\begin{aligned}
x\left(\varphi_{1} \varphi_{2}\right) T_{a} & =\left(a \varphi_{1} \varphi_{2}\right)^{-1}(a x) \varphi_{1} \varphi_{2}=\left(a \varphi_{1} \varphi_{2}\right)^{-1}\left(a \varphi_{1}\left(a \varphi_{1}\right)^{-1}(a x) \varphi_{1}\right) \varphi_{2}= \\
& =\left(\left(a \varphi_{1}\right) \varphi_{2}\right)^{-1}\left(a \varphi_{1}\left(\left(a \varphi_{1}\right)^{-1}(a x) \varphi_{1}\right)\right) \varphi_{2}=x \varphi_{1} T_{a} \cdot \varphi_{2} T_{a \varphi_{1}},
\end{aligned}
$$

или $\left(\varphi_{1} \varphi_{2}\right) T_{a}=\varphi_{1} T_{a} \cdot \varphi_{2} T_{a \varphi_{1}}$, откуда по индукции получим и $(6)$.

Наконец, по (4)

$$
\begin{aligned}
\left(x \varphi T_{a_{1}}\right) T_{a_{2}} & =\left(\left(a_{1} \varphi\right)^{-1}\left(a_{1} x\right) \varphi\right) T_{a_{2}}=\left(\left(a_{1} \varphi\right)^{-1}\left(a_{1} a_{2}\right) \varphi\right)^{-1}\left(a_{1} \varphi\right)^{-1}\left(a_{1} a_{2} x\right) \varphi= \\
& =\left(\left(a_{1} a_{2}\right) \varphi\right)^{-1}\left(a_{1} a_{2} x\right) \varphi=x \varphi T_{a_{1} a_{2}},
\end{aligned}
$$

т. е. и (7) имеет место.

Назовем группу е-отображений группы $A$, отображающую в себя при транслировании всеми элементами группы $A, T$-з а м к н у то й группой отображений группы $A$. Очевидно, группа всех $e$-отображений и все подгрулпы группы автоморфизмов группы $A$ являются ее $T$-замкнутыми группами отображений. Менее тривиальные примеры $T$-замкнутых групп отображений приведены в следующих предложениях.

Если А конечная группа нечетного порядка, то знакопеременная подгруппа симметрической группь всех е-отображений группы $A$ T-замкнута.

Действительно, трансляция $e$-отображения $\varphi$, т. е. отображение $x \leftrightarrow(a \varphi)^{-1}(a x) \varphi$ можно рассматривать как произведение трех подстановок $x \longleftrightarrow a x, x \longleftrightarrow x \varphi$ и $x \leftrightarrow \rightarrow(a \varphi)^{-1} x$ множества всех элементов группы $A$. Так как порядок любого элемента $а$ группы нечетного порядка есть число нечетное, то подстановка $x \longleftrightarrow \rightarrow$ ax четная, потому что ее можно разложить на произведение независимых циклов нечетного порядка $\left(y, a y, \ldots, a^{n-1} y\right)$. По той же причине $x \leftrightarrow(a \varphi)^{-1} x$ является четной подстановкой. Если и $\varphi-$ четная подстановка, то $\varphi T_{a}$ как произведение трех четных подстановок сама четная.

Множество всех е-отображений группь $A$, которье отображают смежные классы группы А по нормальному делителю $Н$ в себя, является T-замкнутой группой отображений группь $A$.

Действительно, множество Ф всех таких $e$-отображений образует группу. Если $\varphi \in \Phi$, то $(a x) \varphi \in a x H, a \varphi \in a H$ и $(a \varphi)^{-1} \in H^{-1}$. Следовательно, $x \varphi T_{a}=(a \varphi)^{-1}(a x) \varphi \in a^{-1} \cdot a x H=x H$, т. е. $\varphi T_{a} \in \Phi$.

Т е ор е м. Все косые произведения двух групп, одним множителем которых является данная группа $А$ и второй множитель которых не содержит нетривиального нормального делителя произведения, , получаются, если для каждой $T$-замкнутой группь отображений Ф группь $A$ составить множество всех пар $(a, \varphi)$, где $a \in A$ и $\varphi \in \Phi, ~ и$ определить на этом множестве операцию равенством

$$
\left(a_{1}, \varphi_{1}\right)\left(a_{2}, \varphi_{2}\right)=\left(a_{1} \cdot a_{2} \varphi_{1}^{-1},\left(\varphi_{1}^{-1} T_{a_{2}}\right)^{-1} \varphi_{2}\right) .
$$

2 ENSV TA Toimetised T-4 60. 
Д ок аз а тельство. Докажем, что операция (8) определяет группу. Так как соответствия $a_{2} \leftrightarrow \rightarrow a_{2} \varphi_{1}^{-1}$ и $\varphi_{1} \leftrightarrow\left(\varphi_{1}^{-1} T_{a_{2}}\right)^{-1}$ взаимно однозначны, то для этого достаточно доказать, что выполнены (2) и (3), где $a^{b}=a \varphi^{-1}$ и $b^{a}=\left(\varphi^{-1} T_{a}\right)^{-1}$. Имеем

$$
\left(a^{b_{1}}\right)^{b_{2}}=\left(a \varphi_{2}^{-1}\right) \varphi_{2}^{-1}=a\left(\varphi_{1}^{-1} \varphi_{2}^{-1}\right)=a\left(\varphi_{2} \varphi_{1}\right)^{-1}=a^{b_{2} b_{1}}
$$

и, пользуясь (7),

$$
\begin{aligned}
\left(b^{a_{1}}\right)^{a_{2}} & =\left(\left(\left(\varphi^{-1} T_{a_{1}}\right)^{-1}\right)^{-1} T_{a_{2}}\right)^{-1}=\left(\left(\varphi^{-1} T_{a_{1}}\right) T_{a_{2}}\right)^{-1}= \\
& =\left(\varphi^{-1} T_{a_{1} a_{2}}\right)^{-1}=b^{a_{1} a_{2}},
\end{aligned}
$$

дальше по (4)

$$
\begin{aligned}
\left(a_{1} a_{2}\right)^{b} & =\left(a_{1} a_{2}\right) \varphi^{-1}=a_{1} \varphi^{-1}\left(a_{1} \varphi^{-1}\right)^{-1}\left(a_{1} a_{2}\right) \varphi^{-1}= \\
& =a_{1} \varphi^{-1} \cdot a_{2} \varphi^{-1} T_{a_{1}}=a_{1} \varphi^{-1} \cdot a_{2}\left(\left(\varphi^{-1} T_{a_{1}}\right)^{-1}\right)^{-1}=a_{1}^{b} a_{2}^{b a_{1}}
\end{aligned}
$$

и по $(6)-$

$$
\begin{aligned}
\left(b_{1} b_{2}\right)^{a}= & \left(\left(\varphi_{1} \varphi_{2}\right)^{-1} T_{a}\right)^{-1}=\left(\left(\varphi_{2}^{-1} \varphi_{1}^{-1}\right) T_{a}\right)^{-1}=\left(\varphi_{2}^{-1} T_{a} \cdot \psi_{1}^{-1} T_{a \varphi_{2}-1}\right)^{-1}= \\
& =\left(\varphi_{1}^{-1} T_{a \varphi_{2}^{-1}}\right)^{-1}\left(\varphi_{2}^{-1} T_{a}\right)^{-1}=b_{1}^{a^{b_{2}}} b_{2}^{a},
\end{aligned}
$$

так, что (2) и (3) выполнены.

Определенная операцией (8)_группа $G$ является, очевидно, косым произведением своих подгрупп $\bar{A}$ и $\Phi$, образованных всеми парами вида $(a, \varepsilon)$ и $(e, \varphi)(\varepsilon-$ единица группы $\Phi)$ соответственно. Подгруппа $\bar{A}$ изоморфна группе $A$. Подгруппа $\Phi$ не содержит нетривиального нормального делителя группы $G$, так как совокупность всех таких $\bar{\varphi} € \bar{\Phi}$, для которых $a^{b}=a \varphi^{-1}=a$ при любом $a \in A$, является, по [3], максимальным нормальным делителем $G$, содержащимся в $\bar{\Phi}$, а в рассматриваемом случае эта совокупность состоит только из единицы группы $Ф$.

Пусть теперь $A \circ B-$ некоторое косое произведение, в котором множитель $B$ не содержит нетривиального нормального делителя произведения. Тогда можно $A \circ B$ рассматривать как множество пар $[a, b]$, на котором определена операция равенством (1), причем выполнены (2) и (3). Обозначим $a^{b}=a \varphi$. Тогда $\varphi$ является $e$-отображением группы $A$. Действительно, $\varphi$ взаимно однозначно и если в (2) взять $a_{1}=a, a_{2}=e$, то $a^{b}=a^{b} e^{b^{a}}$, откуда $e^{b^{a}}=e$, или $e \varphi=e$, так как $b^{a}$ пробегает вместе c $b$ всю группу $B$. Соответствие $b \rightarrow \varphi$ отображает вследствие (2) группу $B$ антигомоморфно на некоторую подгруппу $\Phi$ группы всех $e$-отображений группы $A$. Ядро этого гомоморфизма, как максимальный нормальный делитель группы $A{ }^{\circ} B$, содержащийся в $B$ (по [3]), равно, по нашему условию, единице. Таким образом, $B$ изоморфна Ф. Покажем, что Ф $T$-замкнута. Действительно, если $x \varphi=x^{b}$, то, по (3), имеем

$$
x \varphi T_{a}=(a \varphi)^{-1}(a x) \varphi=\left(a^{b}\right)^{-1}(a x)^{b}=\left(a^{b}\right)^{-1} a^{b} x^{b^{a}}=x^{b^{q}},
$$

так что $\varphi T_{a}$ как образ элемента $b^{a}$ при соответствии $b \rightarrow \varphi$ содержится в Ф при любом $a \in A$.

Образуем теперь множество $G$ всех пар $(a, \varphi)$, где $a \in A$ и $\varphi \in \Phi$ и определим на этом множестве операцию равенством (8). Соответствие $[a, b] \rightarrow\left(a, \varphi^{-1}\right)$ отображает $A \circ B$ изоморфно на $G$. Действительно, это 
соответствие взаимно однозначно, и произведению $\left[a_{1}, b_{1}\right]\left[a_{2}, b_{2}\right]=$ $=\left[a_{1} a_{2}^{b_{1}}, b_{1}^{a_{2}} b_{2}\right]$ соответствует пара $g=\left(a_{1} \cdot a_{2} \varphi_{1},\left(\varphi_{1} T_{a_{2}}\right)^{-1} \varphi_{2}^{-1}\right)$, так как $a_{2}^{b_{1}}=a_{2} \varphi_{1}$ и по (2) и (4)

$$
\begin{aligned}
a^{b_{1}^{a_{2}} b_{2}} & =\left(a^{b_{2}}\right)^{b_{1}^{a_{2}}}=\left(a_{2}^{b_{1}}\right)^{-1}\left(a_{2}^{b_{1}}\left(a^{b_{2}}\right)^{b_{1}^{a_{2}}}\right)=\left(a_{2}^{b_{1}}\right)^{-1}\left(a_{2} a^{b_{2}}\right)^{b_{1}}= \\
& =\left(a_{2} \varphi_{1}\right)^{-1}\left(a_{2} \cdot a \varphi_{2}\right) \varphi_{1}=a \varphi_{2}\left(\varphi_{1} T_{a_{2}}\right) .
\end{aligned}
$$

Но по (8) $\left(a_{1}, \varphi_{1}^{-1}\right)\left(a_{2}, \varphi_{2}^{-1}\right)=\left(a_{1} \cdot a_{2} \varphi_{1},\left(\varphi_{1} T_{a_{9}}\right)^{-1} \varphi_{2}^{-1}\right)=g$. Теорема доказана.

Если Ф и две Т-замкнутые группь отображений группь $А$ и найдется такой автоморфизм а группы $A$, что $\Psi=\alpha^{-1} \Phi \alpha$, то соответствующие косые произведения, построенные по теореме, изоморфны.

Действительно, соответствие $(a, \varphi) \rightarrow(a \alpha, \psi)=\left(a \alpha, \alpha^{-1} \varphi \alpha\right) \quad$ является изоморфизмом между $A \circ \Phi$ и $A \circ \Psi$, так как по $(8),(6)$ и вследствие того, что $\alpha$ и $\alpha^{-1}$ как автоморфизмы группы $A$ оставляются на месте при трансляции,

$$
\begin{aligned}
\left(a_{1} \alpha, \alpha^{-1} \varphi_{1} \alpha\right)\left(a_{2} \alpha, \alpha^{-1} \varphi_{2} \alpha\right) & =\left(a_{1} \alpha \cdot a_{2} \alpha \alpha^{-1} \varphi_{1}^{-1} \alpha,\left(\left(\alpha^{-1} \varphi_{1}^{-1} \alpha\right) T_{a_{2} \alpha}\right)^{-1} \alpha^{-1} \varphi_{2} \alpha\right)= \\
& =\left(a_{1} \alpha \cdot a_{2} \varphi_{1}^{-1} \alpha,\left(\alpha^{-1} \cdot \varphi_{1}^{-1} T_{a_{2} \alpha \alpha^{-1}} \cdot \alpha\right)^{-1} \alpha^{-1} \varphi_{2} \alpha\right)= \\
& =\left(\left(a_{1} \cdot a_{2} \varphi_{1}^{-1}\right) \alpha, \alpha^{-1}\left(\varphi_{1}^{-1} T_{a_{2}}\right)^{-1} \varphi_{2} \alpha\right) .
\end{aligned}
$$

Л ИТ Е Р А У Р А

1. G. Z a p pa, Atti secondo Congresso Un. Mat. Ital. Bologna 1940, Roma, 1942 . $119-125$.

2. J. Dougla s, Proc. Nat. Acad. Sci. USA, 37, 1951, 604-610.

3. J. S z é p, Comment. Math. Helv., 22, 1948, 31-33.

$\begin{array}{cc}\text { Институт кибернетики } & \text { Поступила в редакцию } \\ \text { Академии наук Эстонской ССР } & 29 \text { XII } 1959\end{array}$

\section{KAHE VAHETATAVA RUHMA KORRUTISTE KONSTRUKTSIOONIST}

\section{Petersen}

Resümee

Artiklis käsitletakse kahe vahetatava rühma $A$ ja $B$ selliseid korrutisi $(G=A \circ B)$, milles iga elementi vōib esitada üheselt kujul $g=a b$ ning mille tegur $B$ ei sisalda korrutise mittetriviaalset normaaljagajat. Tōestatakse, et iga niisugust korrutist vöib antud esimese teguri $A$ puhul konstrueerida paaride $(a, \varphi)$ hulgana, millel operatsioon on defineeritud võrdusega (8), kus $a \in A, \varphi \in \Phi$ ja $\Phi$ on rühma $A$ kõigi üks-üheste ühikut paigale jätvate iseeneselekujutuste rühma alarühm, mis on kinnine kõigi vôrdusega (4) defineeritud operatsioonide $T_{a}(a \in A)$ suhtes. 


\section{UBER DIE KONSTRUKTION DER PRODUKTE ZWEIER VERTAUSCHBARER GRUPPEN}

\section{Petersen}

\section{Zusammenjassung}

In der Abhandlung werden solche Produkte $G=A \circ B$ vertauschbarer Gruppen $A$ und $B$ betrachtet, bei denen jedes Element eindentig in der Form $g=a b$ darstellbar ist und deren Teiler $B$ keinen nichttriviellen Normalteiler des Produktes enthält. Es wird nachgewiesen, dass jedes Produkt dieser Art mit gegebenem erstem Teiler $A$ sich als eine Menge von Paren ( $a, \varphi)$ durch die Operation (8) konstruieren lässt, wo $a \in A$, $\varphi \in \Phi$ und $\Phi$ eine Untergruppe der Gruppe aller ein-eindeutigen Abbildungen der Gruppe $A$ an sich ist, die das Einzelelement invariant lassen, wobei $\Phi$ bezüglich aller durch (4) definierten Operationen $T_{a}(a \in A)$ abgeschlossen ist.

\section{Institut für Kybernetik}

der Akademie der Wissenschaften dei Estnischen SSR
Eingegangen

am 29. Dez. 1959 